\title{
A Radiation Oncologist's Story: High Tech Meets High Touch
}

\author{
Richard S. Pieters, MD, MEd, FACR
}

I GREW UP HEARING STORIES ABOUT MY GRANDMOTHER, a missionary and an American-trained surgeon, who brought her doctoring skills to Korea at the turn of the twentieth century. She spent her first few months there learning the language and customs of this ancient kingdom where she'd spend the rest of her life. Working in a preantibiotic era, supportive care was an important part of her practice. Keeping her patients comfortable was sometimes the best she could offer (see Fig. 1). The personal side of medicine, often known as high touch, was as important as her surgical technique. Thus began my appreciation of the interplay between high tech and high touch.

Not surprisingly, as an undergraduate I majored in anthropology and East Asian studies. When later accepted to medical school, I saw myself as a family practitioner. A chance conversation with a radiation oncologist on a shared car ride and a subsequent elective in the field changed my career course. Radiation oncology seemed the perfect way to merge some of my major life influences. I would be able to enlist my math and physics skills, courtesy of my math tea- cher father, into the service of hands-on doctoring like my grandmother, or so I thought.

Radiation oncology has long been on the leading edge of technological advances in treatment planning and delivery systems. During my residency, computerized tomography (CT) imaging became more readily available. Today, CT-based volumetric planning with the fusion of magnetic resonance imaging (MRI) and positron-emitting tomography (PET) scans, automated beam-shaping devices, and daily field imaging are common in practice. In plain language, with their current tools, radiation oncologists can customize treatment to accurately target the tumor and better spare surrounding normal tissue.

During my residency, rigorous attention was given to mastery of the technical aspects of radiation oncology, but supportive care, though deemed important, was never given the same intense focus. You learned how to address treatmentrelated side effects on a case-by-case basis. Instruction and feedback on communication skills were not formalized and rarely mentioned, except when a major faux pas occurred

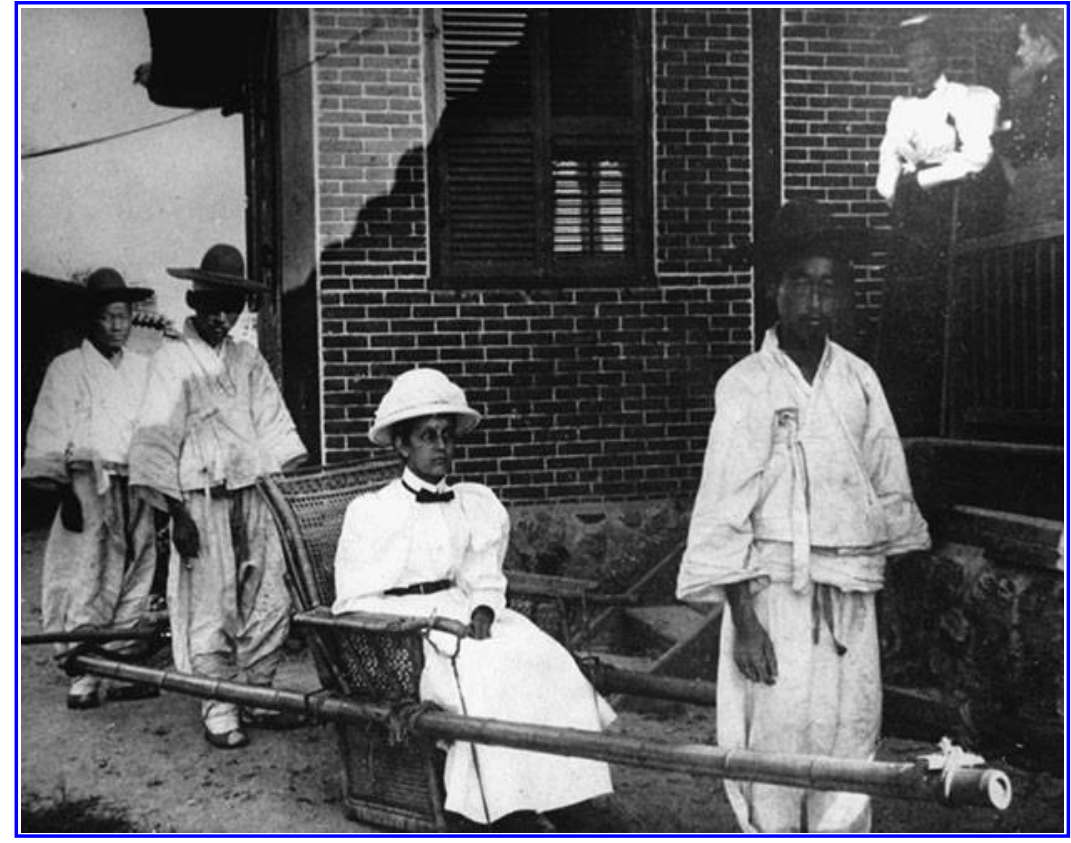

FIG. 1. My grandmother leaving for a house call, circa 1897, (@) Richard S. Pieters, Published with permission.)

Department of Radiation Oncology, University of Massachusetts Medical School, Worcester, Massachusetts. 
when talking with a patient. The prevailing wisdom of the time was either you had communication talents or you didn't. ${ }^{1}$

Early in my attending career, I was assigned a large pediatric caseload at a comprehensive cancer center. Because children are growing, they are particularly vulnerable to the effects of radiation. They are also at risk for late effects, such as a second cancer, decades after treatment. Therefore, informed consent discussions on the risks versus benefits of radiation treatment are important. During the initial consultation, parents sometimes told me they were hearing the word "cancer" rather than "growth" or "tumor" for the first time. This sudden revelation could provoke distress for patient and doctor. I quickly learned I had to gently probe for how much they knew and gauge how much to share without becoming too overwhelming. At times, it was a delicate balancing act.

As my career went on, I developed clinical and research interests in supportive care, late effects, and the palliative uses of radiation therapy. And then, a few years ago, another chance meeting occurred. A palliative care service was starting at the hospital and its multidisciplinary team meetings were held in a conference room adjacent to my department. I began to attend as regularly as I could. When a practice pathway to certification in hospice and palliative medicine through a mini-fellowship program became available, I decided to "grandfather" before the opportunity closed.

Given my clinical commitments, the requirement of 100 hours of participation on a multidisciplinary team presented a challenge. I used vacation and academic meeting time to visit other institutions with training programs in hospice and palliative medicine. I was very fortunate to receive invitations to spend one week at an inpatient palliative care unit and two weeks at a hospice training program. As a physician in my 30th postgraduate year (PGY-30), it was humbling to find myself shadowing the treatment team. However, it was also rewarding to have the luxury to spend uninterrupted blocks of time with individual patients and listen to their stories.
Through this late-in-career training, I dramatically improved my communication and supportive care skills, particularly in pain management. Dr. Robert Buckman, a medical oncologist and communication expert, states, "Effective symptom control is impossible without effective communication."1 Pain management and communication techniques, such as the SPIKES (setting, perception, invitation, knowledge, emotions, strategy) protocol, ${ }^{1}$ were formally taught to me for the first time in my career. It was professionally gratifying to learn both the art and science of these essential skills, particularly breaking difficult or bad news. Basic competencies in palliative care, such as pain management and communication on prognosis and treatment goals, should be incorporated into the training of all physicians. $^{2}$

I know that this experience has made me a better doctor and a stronger radiation oncologist. I feel I have finally come full circle. I remain grateful to colleagues who opened doors and shared knowledge. At long last, I have found balance in the dynamic interplay between high tech and high touch in my chosen field. I hope my grandmother would be pleased.

\section{References}

1. Buckman R: Communication skills in palliative care: A practical guide. Neurol Clin 2001;19:989-1004.

2. Quill TE, Abernethy AP: Generalist plus specialist palliative care: Creating a more sustainable model. New Engl J Med 2013;368:1173-1175.

Address correspondence to: Richard S. Pieters, MD, MEd, FACR Department of Radiation Oncology UMass Memorial Medical Center 55 Lake Avenue, North Worcester, MA 01655

E-mail: rpieters@massmed.org 